\title{
DIRECT INTERFERENCE SUPPRESSION IN EEG/MEG DIPOLE SOURCE LOCALIZATION
}

\author{
Shun Chi Wu, A. Lee Swindlehurst and Yu Chen Yao \\ University of California, Irvine \\ Department of Electrical Engineering and Computer Science \\ Irvine, CA 92697, USA
}

\begin{abstract}
An interference suppression algorithm is proposed for canceling the spatially correlated background noise and interference in EEG/MEG source localization applications. Rather than using the standard prewhitening approach, the proposed algorithm attempts to directly null interference using a projection operator obtained from a set of secondary, control-state data. The use of direct interference cancellation is shown to be significantly more robust than prewhitening, due to the elimination of the need for computing covariance matrices, and the relaxation of the assumption of temporal stationarity. Simulation examples are presented to demonstrate the robust performance of the algorithm.
\end{abstract}

Index Terms - EEG, MEG, interference suppression.

\section{INTRODUCTION}

Techniques such as electroencephalography (EEG) and magnetoencephalography (MEG) are non-invasive brain imaging methods that provide direct measurement of cortical activity with high temporal resolution, and these techniques are widely used in the study of brain activity. In many applications, an important function of the EEG/MEG measurements is to determine, with as great a precision as possible, the location of the source of brain activity evoked by a given stimulus. This requires a large array of sensors or electrodes, and leads to be a high-dimensional inverse problem that in general does not have a unique solution. To tackle this problem, a "forward" propagation model for the brain and skull region is adopted, and then one attempts to estimate the parameters of the model corresponding to the source activity. It is common for the EEG/MEG neural activity in a small region of the brain to be modeled as equivalent current dipoles, with associated position, dipole orientation and dipole moment magnitude as parameters to be estimated [1].

High resolution techniques for estimating the parameters of source dipoles have already been proposed, including methods based on MUSIC [2, 3] and linearly constrained beamforming (LCMV) [4]. Such methods cannot be directly applied in practice due to the fact that the signals of inter- est are very weak, and buried in strong, spatially correlated background noise and interference due primarily to background brain activity not related to the stimulus and noise in the sensors and associated electronics [5]. The presence of this unmodeled interference will degrade the performance of MUSIC or LCMV to the point that the resulting parameter estimates are virtually useless.

Consequently, existing approaches [5, 6] use a set of "control state" measurements taken prior to the application of the stimulus in order to estimate the statistics of the nominal background interference and noise. In particular, the control state data is used to estimate the covariance matrix of the interference plus noise, which in turn is used to prewhiten the data taken after the stimulus is applied, or in other words, during the "task state." There are two primary drawbacks to this approach: (1) estimation of a large covariance matrix typically requires collecting a large amount of data (typically two to three times as many data samples as there are sensors in the array), and (2) the use of prewhitening presupposes that the noise and interference are stationary in both space and time. If these conditions are not met, then significant performance degradations can occur.

In this paper, we present an alternative "direct" approach for interference suppression. Instead of using the control state measurements to estimate the covariance matrix for whitening the task state data prior to localization, the measurements are used to construct a subspace that is (approximately) orthogonal to the interference. Localization can then performed by a standard technique after projecting the task state measurements onto this subspace. This approach is a special case of the autoregressive filtering technique of [7], since it relies only on the stationarity of the spatial parameters of the background interference. As we will see later, the performance of this approach is superior to techniques based on prewhitening, especially in situations where the signal to interference ratio (SIR) is small. 


\section{STANDARD DATA MODEL AND ASSUMPTIONS}

\subsection{Standard Data Model}

Following the mathematical model of $[3,4,5]$, the outputs of $m$ sensors (electrodes, detector coils or both) sampled at some time instant $t$ are stacked into a $m \times 1$ vector $\mathbf{x}(t)$. If there are $N_{S}$ dipoles present (i.e. the sources of interest or SoI) when a given stimulus is applied, the output of the array may be written as a linear superposition of the signals from each source:

$$
\mathbf{x}(t)=\mathbf{A s}(t)+\mathbf{n}(t),
$$

where the $N_{S} \times 1$ signal vector $\mathbf{s}(t)$ is composed of the moment magnitudes of $N_{S}$ dipoles sampled at time $t$. The term $\mathbf{n}(t)$ is composed of both background electronic/sensor noise and contributions from interfering EEG/MEG sources that are non-stimulus-related and therefore not of interest. The matrix $\mathbf{A}=\left[\mathbf{a}_{1}, \mathbf{a}_{2}, \ldots, \mathbf{a}_{N_{S}}\right]$ is comprised of $N_{S}$ vectors of dimension $m \times 1$ that correspond to the lead field vectors (LFV) of each dipole source. The $k^{\text {th }} \mathrm{LFV} \mathbf{a}_{k}\left(\mathbf{r}_{k}, \phi_{k}\right)$ represents the response of the sensor array to a dipole located at position $\mathbf{r}_{k}$ with dipole orientation denoted by $\phi_{k}=\left[\phi_{x k}, \phi_{y k}, \phi_{z k}\right]$. In practice, a LFV model can be derived from multilayer spherical head models $[1,3]$ or based on realistic head models from computed tomography or magnetic resonance imaging data [8].

The LFV model is typically decomposed into three parts, each due to a separate orientation component:

$$
\begin{aligned}
\mathbf{a}_{k}\left(\mathbf{r}_{k}, \phi_{k}\right) & =\mathbf{G}\left(\mathbf{r}_{k}\right) \phi_{k} \\
& =\mathbf{g}_{x}\left(\mathbf{r}_{k}\right) \phi_{x k}+\mathbf{g}_{y}\left(\mathbf{r}_{k}\right) \phi_{y k}+\mathbf{g}_{z}\left(\mathbf{r}_{k}\right) \phi_{z k},
\end{aligned}
$$

where $\mathbf{g}_{x}\left(\mathbf{r}_{k}\right), \mathbf{g}_{y}\left(\mathbf{r}_{k}\right)$ and $\mathbf{g}_{z}\left(\mathbf{r}_{k}\right)$ are the LFV's that result from unit amplitude dipole sources oriented in the $\mathrm{x}, \mathrm{y}$ and $\mathrm{z}$ directions respectively. It is well known that, due to the fact that response of the $m$-sensor system is a linear function of the orientation components, it is possible to decouple the estimation of locations and orientations and find a more efficient solution instead of search for all parameters simultaneously.

\subsection{Assumptions}

The following assumptions are made in this paper:

A1 The number of interfering sources plus the number of SoI's is less than the number of sensors: $N_{I}+N_{S}<m$.

A2 Every set of $p<m$ lead field vectors is linearly independent.

A3 The source signals from different dipoles are linearly independent.

A4 The background electronic/sensor noise is spatially and temporally white with zero mean and variance $\sigma^{2}$.

Assumptions A2-A4 are standard for existing approaches, while A1 is typically less stringent for methods based on prewhitening: $N_{S}<m$. Practically, however, prewhitening methods will not perform well if $N_{I}+N_{S}<m$ does not hold. In most applications of interest, $m$ is large enough and the number of strong interferers is small enough for A1 to be reasonable.

\section{LOCALIZATION ALGORITHMS}

We briefly review here the MUSIC and LCMV approaches for dipole localization based on prewhitening. These approaches require estimation of the covariance matrix of the control state data $\mathbf{x}_{C}(t)$, which is typically achieved as follows

$$
\hat{\mathbf{R}}_{C}=\frac{1}{n} \sum_{t=1}^{n} \mathbf{x}_{C}(t) \mathbf{x}_{C}^{T}(t),
$$

where $n$ is the number of control state samples. As mentioned earlier, to obtain a reliable estimate of $\mathbf{R}_{C}$ requires at least $n>2 m$ samples. The task state data, $\mathbf{x}_{T}(t)$, is then prewhitened as follows:

$$
\mathbf{x}_{T}^{\prime}(t)=\hat{\mathbf{R}}_{C}^{-1 / 2} \mathbf{x}_{T}(t),
$$

after which the covariance matrix of the prewhitened task state data, $\hat{\mathbf{R}}_{T}^{\prime}$, is estimated as in (3). For the prewhitening to be effective, one must assume that the spatial and temporal statistics of the interference are stationary over the control and task state measurement periods.

\subsection{MUltiple SIgnal Calssification (MUSIC)}

MUSIC [2, 3] is based on calculation of the "noise subspace," which is defined to be the $m-N_{S}$ eigenvectors of $\hat{\mathbf{R}}_{T}^{\prime}$ with the smallest eigenvalues. Collecting these eigenvectors in the matrix $\mathbf{E}_{N}$, MUSIC locates the dipoles as those whose LFV's are most orthogonal to $\mathbf{E}_{N}$ :

$$
V_{\mathrm{MU}}(\mathbf{r}, \boldsymbol{\phi})=\frac{\phi^{T} \mathbf{G}^{T}(\mathbf{r}) \mathbf{E}_{N} \mathbf{E}_{N}^{T} \mathbf{G}(\mathbf{r}) \boldsymbol{\phi}}{\phi^{T} \mathbf{G}^{T}(\mathbf{r}) \mathbf{G}(\mathbf{r}) \boldsymbol{\phi}}
$$

Since $\phi$ is unconstrained (except for $\phi \neq 0$ ), minimizing $V_{M U}(\mathbf{r}, \phi)$ with respect to $\phi$ is equivalent to a 3 -dimensional generalized eigenvalue search:

$$
\begin{gathered}
\min _{\mathbf{r}} \lambda_{\min }(\mathbf{r}) \\
\mathbf{G}^{T}(\mathbf{r}) \mathbf{E}_{N} \mathbf{E}_{N}^{T} \mathbf{G}(\mathbf{r}) \boldsymbol{\phi}=\lambda_{\min }(\mathbf{r}) \mathbf{G}^{T}(\mathbf{r}) \mathbf{G}(\mathbf{r}) \boldsymbol{\phi} .
\end{gathered}
$$

The position estimates are found by viewing $\lambda_{\min }$ as a function of $\mathbf{r}$ and then searching for its minima. The orientation of the dipole with location estimate $\hat{\mathbf{r}}$ is then taken to be the eigenvector associated with $\lambda_{\min }(\hat{\mathbf{r}})$.

\subsection{Linearly Constrained Beamforming (LCMV)}

The LCMV [4] approach locates the dipoles through "beams" formed by providing unit gain at each possible source position 
and minimizing array output power from all other locations. The solution has a form similar to that of MUSIC:

$$
\begin{gathered}
\min _{\mathbf{r}} \lambda_{\min }(\mathbf{r}) \\
\mathbf{G}^{T}(\mathbf{r}) \hat{\mathbf{R}}_{T}^{\prime-1} \mathbf{G}(\mathbf{r}) \boldsymbol{\phi}=\lambda_{\min }(\mathbf{r}) \mathbf{G}^{T}(\mathbf{r}) \mathbf{G}(\mathbf{r}) \boldsymbol{\phi} .
\end{gathered}
$$

Position and orientation estimates are found in the same fashion as in MUSIC.

\section{INTERFERENCE SUPPRESSION USING NULL SPACE PROJECTION}

In this section, we propose an alternative way to suppress the interference. Instead of using the control state measurements to estimate the covariance matrix of the interference for whitening the task state measurements prior to localization, the measurements are used to construct a subspace that is orthogonal to the interference. The data models used here are

$$
\begin{aligned}
\text { Control }: \mathbf{X}_{C} & =\mathbf{A}_{I} \mathbf{S}_{I C}+\mathbf{W}_{C}, \\
\text { Task }: \mathbf{X}_{T} & =\mathbf{A}_{S} \mathbf{S}_{S}+\mathbf{A}_{I} \mathbf{S}_{I T}+\mathbf{W}_{T},
\end{aligned}
$$

where $\mathbf{X}_{C}=\left[\mathbf{x}_{C}(1), \ldots, \mathbf{x}_{C}(n)\right]$ is the matrix of measured control state data vectors, and $\mathbf{X}_{T}$ is defined similarly. The additive noise is explicitly broken down into an interference $\mathbf{A}_{I} \mathbf{S}_{I}(t)$ and noise term $\mathbf{W}$. The matrices $\mathbf{A}_{S}$ and $\mathbf{A}_{I}$ are the lead field matrices of the SoI's and the interference, respectively. In practice, due to the presence of $\mathbf{W}_{C}, \mathbf{X}_{C}$ will be full rank and thus will have no true null space. A reasonable approach to find the solution is to use the following least squares criterion to estimate the $m \times m-N_{I}$ null space $\mathbf{H}$ [7]:

$$
\hat{\mathbf{H}}=\arg \min _{\mathbf{H}}\left\|\mathbf{H}^{T} \mathbf{X}_{C}\right\|_{F}^{2} .
$$

Assuming $\mathbf{H}^{T} \mathbf{H}=\mathbf{I}$ to avoid a trivial solution and defining the decomposition of the control state measurements as

$$
\mathbf{X}_{C}=\mathbf{U} \Sigma \mathbf{V}^{T}=\left[\begin{array}{ll}
\mathbf{U}_{I} & \mathbf{U}_{N}^{C}
\end{array}\right]\left[\begin{array}{cc}
\Sigma_{I} & 0 \\
0 & \Sigma_{N}
\end{array}\right] \mathbf{V}^{T},
$$

the solution of (9) will be $\hat{\mathbf{H}}=\mathbf{U}_{N}^{C}$, the $m-N_{I}$ left singular vectors of $\mathbf{X}_{C}$ with smallest singular values. The projection matrix onto this null space can be written as $\mathbf{P}_{H}=\hat{\mathbf{H}} \hat{\mathbf{H}}^{T}$. Projecting the task state measurements onto this null subspace, we have

$$
\mathbf{P}_{H} \mathbf{X}_{T}=\mathbf{P}_{H} \mathbf{A}_{S} \mathbf{S}_{S}+\mathbf{P}_{H} \mathbf{W}_{T},
$$

which in principle contains only the SoI's and the electronic/sensor noise.

The interference projection alters the lead field matrix of the desired sources, so MUSIC and LCMV must be modified prior to implementation. If we define $\mathbf{Y}_{T}=\mathbf{P}_{H} \mathbf{X}_{T}$ and $\mathbf{G}^{\prime}=\mathbf{P}_{H} \mathbf{G}$, and use the following singular value decomposition:

$$
\mathbf{Y}_{T}=\mathbf{U}^{\prime} \Sigma^{\prime} \mathbf{V}^{\prime T}=\left[\mathbf{U}_{S}^{\prime} \mathbf{U}_{N}^{\prime}\right]\left[\begin{array}{cc}
\Sigma_{S}^{\prime} & 0 \\
0 & \Sigma_{N}^{\prime}
\end{array}\right] \mathbf{V}^{\prime T},
$$

the modified MUSIC approach becomes

$$
\begin{gathered}
\min _{\mathbf{r}} \lambda_{\min }(\mathbf{r}) \\
\mathbf{G}^{\prime T}(\mathbf{r}) \mathbf{U}_{N}^{\prime} \mathbf{U}_{N}^{\prime}{ }^{T} \mathbf{G}^{\prime}(\mathbf{r}) \boldsymbol{\phi}=\lambda_{\min }(\mathbf{r}) \mathbf{G}^{\prime T}(\mathbf{r}) \mathbf{G}^{\prime}(\mathbf{r}) \boldsymbol{\phi} .
\end{gathered}
$$

We will refer to this approach as "Null Projection" (NP) MUSIC. The NP version of LCMV can be obtained by simply use an estimate $\hat{\mathbf{R}}_{Y}$ of the covariance of $\mathbf{Y}_{T}$, as follows:

$$
\begin{gathered}
\min _{\mathbf{r}} \lambda_{\min }(\mathbf{r}) \\
\mathbf{G}^{\prime T}(\mathbf{r}) \hat{\mathbf{R}}_{Y}^{-1} \mathbf{G}^{\prime}(\mathbf{r}) \boldsymbol{\phi}=\lambda_{\min }(\mathbf{r}) \mathbf{G}^{\prime T}(\mathbf{r}) \mathbf{G}^{\prime}(\mathbf{r}) \boldsymbol{\phi} .
\end{gathered}
$$

\section{SIMULATION EXAMPLES}

\subsection{Simulation Parameters}

Although the proposed algorithms are applicable to both EEG and MEG source localization, we choose an EEG simulation involving 77 electrodes from the 10-10 system described in [9] as an example. The LFV's were calculated using the approximate method of [1], in which the head was modeled as a four-layer sphere with its radii and conductivities specified in [10]. The number of the interference sources was set to 30 , and they were placed randomly on the surface of brain. The signals generated by the interferers were taken from the electrode outputs of real EEG measurements. The control and task state data intervals were assumed to be the same length in time, covering 100 samples. The signal to noise ratio (SNR) was defined as $\left\|\mathbf{A}_{S} \mathbf{S}_{S}\right\|_{F}^{2} /\|\mathbf{W}\|_{F}^{2}$, and similarly the signal to interference ratio (SIR) was defined to be $\left\|\mathbf{A}_{S} \mathbf{S}_{S}\right\|_{F}^{2} /\left\|\mathbf{A}_{I} \mathbf{S}_{I}\right\|_{F}^{2}$. In all of the simulations, we assume that each SoI has unit power and all interference waveforms have the same power which is specified by a given SIR.

\subsection{Results and Discussion}

To compare the performance of the proposed interference suppression approach with the existing prewhitening based approach, a simple simulation study was conducted. In this example, The SNR was set at $15 \mathrm{~dB}$ and the SIR varied from $5 \mathrm{~dB}$ to $-5 \mathrm{~dB}$. Three dipole sources were placed in fixed positions on the surface of brain, near the positions of sensors Fz, $\mathrm{C} 3$ and $\mathrm{C} 4$. The dipole orientations were chosen randomly. Both MUSIC and LCMV were used for estimating positions and orientations of these dipoles.

Figure 1 shows the spatial spectrum (top view only) of the four algorithm combinations for a single experiment. PW stands for "prewhitened" in the figure legend. The white squares represent the true positions of the SoI's, and the black dots represent the locations of the interfering sources. The SIR for this example is $5 \mathrm{~dB}$. As shown in Figure 1, the direct interference cancellation approaches do a significantly better job of removing the effects of the background noise, while the prewhitened approaches still suffer from the influence of the interference (particuarly the PW-LCMV approach). Figures 2 and 3 show the RMS dipole location and orientation errors estimates, respectively, averaged over 30 independent trials(i.e. 90 estimates). We kept the dipole sources near the positions of sensors $\mathrm{Fz}, \mathrm{C} 3$ and $\mathrm{C} 4$ from trial to trial with 


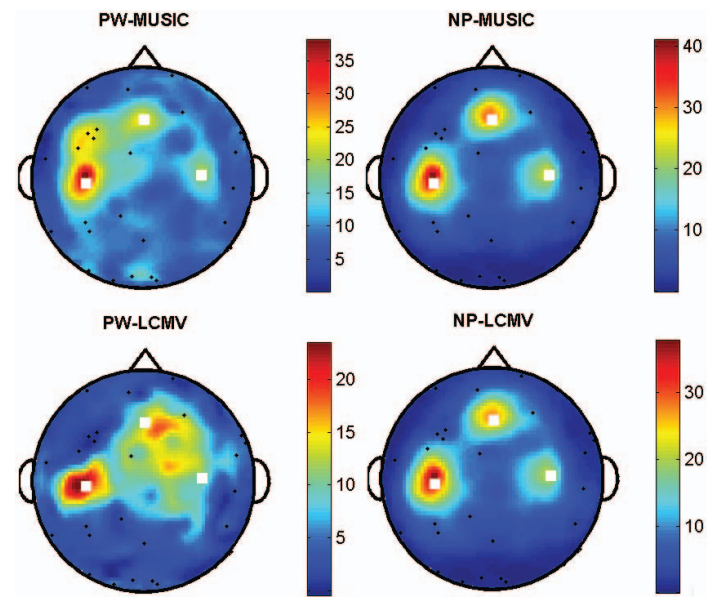

Fig. 1. Spatial spectrum of four algorithm combinations.

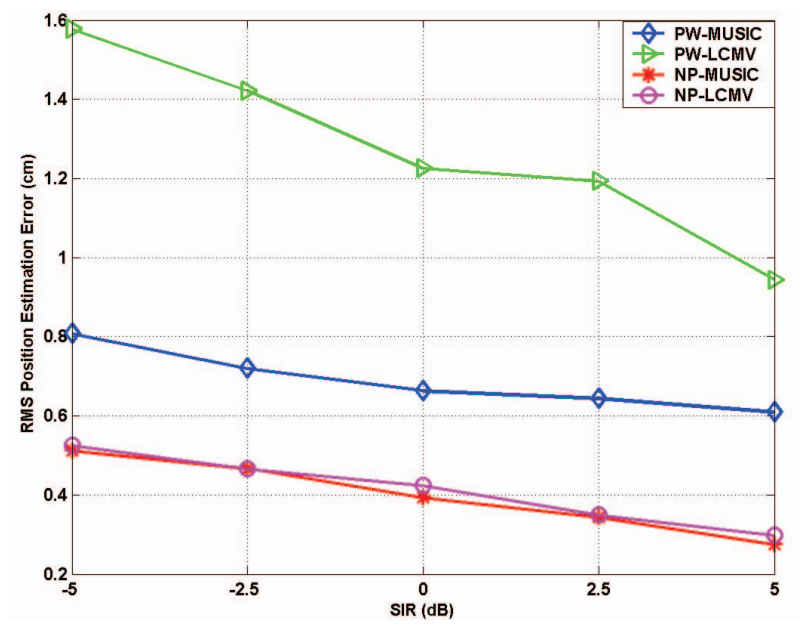

Fig. 2. RMS position estimation errors.

their orientations, the positions and orientations of the interferers being randomly changed. As shown in the figures, the approaches based on prewhitening have significantly larger estimation errors in both location and orientation than the direct interference cancellation methods. Moreover, both NP-MUSIC and NP-LCMV have similar performance.

\section{CONCLUSION}

We have presented an efficient algorithm for the direct suppression of background interference in EEG/MEG source localization applications. The proposed method is based on projecting the task state measurements onto the null space of the interference, which is estimated directly from the control state measurements. This approach is effective in scenarios where the number of measurements is insufficient for accurately estimating the covariance matrices needed for prewhitening. In addition, the proposed method requires no assumption regarding the temporal stationarity of the interference, which provides additional robustness.

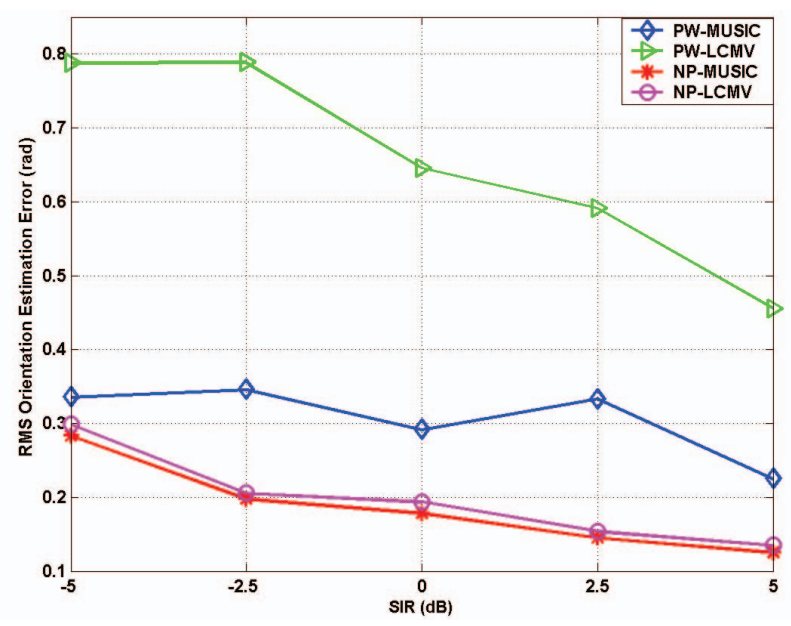

Fig. 3. RMS orientation estimation errors.

\section{REFERENCES}

[1] M. Sun, "An efficient algorithm for computing multi-shell spherical volume conductor models in eeg dipole source localization," IEEE Trans. Biomed. Eng., vol. 44, pp. 1243-1252, 1997.

[2] R. Schmidt, "Multiple emitter location and signal parameter estimation," IEEE Trans. Antenn. and Propagat., vol. 34, pp. 276-280, 1986.

[3] J. C. Mosher, P. S. Lewis, and R. M. Leahy, "Multiple dipole modeling and localization form spatio-temporal meg data," IEEE Trans. Biomed. Eng., vol. 39, pp. 541-557, 1992.

[4] B. D. van Veen, W. van Drongelen, M. Yuchtman, and A. Suzuki, "Localization of brain electrical activity via linearly constrained minimum variance spatial filtering," IEEE Trans. Biomed. Eng., vol. 44, pp. 867-880, 1997.

[5] K. Sekihara, K. Hild, and S. Nagarajan, "A novel adaptive beamformer for meg source reconstruction effective when large background brain activities exist," IEEE Trans. Biomed. Eng., vol. 53, pp. 1755-1764, 2006.

[6] F. Asano, M. Kimura, D. Shibuya, and Y. Kamitani, "Localization and extraction of brain activity using generalized eigenvalue decomposition," ICASSP, pp. 565-568, 2008.

[7] P. Parker and A. Swindlehurst, "Space-time autoregressive filtering for matched subspace stap," IEEE Trans. Aero. and Elec. Sys., vol. 39, pp. 510-520, 2003.

[8] B. N. Cuffin, "Eeg localization accuracy improvements using realistically shaped head models," IEEE Trans. Biomed. Eng., vol. 43, pp. 299-303, 1996.

[9] R. Oostenveld and P. Praamstra, "The five percent electrode system for high-resolution eeg and erp measurements," Clin. Neurophysiol., vol. 112, pp. 713-719, 2001.

[10] B. N. Cuffin and D. Cohen, "Comparison of the magnetoencephalogram and electroencephalogram," Electroencephalogr. Clin. Neurophysiol., vol. 47, pp. 132-146, 1979. 